\title{
Expert Rule Versus Majority Rule Under Partial Information, II
}

DANIEL BEREND $\dagger$

berend@cs.bgu.ac.il

Departments of Mathematics and of Computer Science, Ben-Gurion University, BeerSheva, 84105, Israel

LUBA SAPIR ${ }^{*}$

lsapir@cs.bgu.ac.il

Department of Mathematics, Ben-Gurion University, Beer-Sheva, 84105, Israel

\begin{abstract}
The main purpose of this paper is clarifying the connection between some characteristics of a deciding body and the probability of its making correct decisions. In our model a group of decision makers is required to select one of two alternatives. We assume the probabilities of the decision makers being correct are independent random variables distributed according to the same given distribution rule. This distribution belongs to a general family, containing the uniform distribution as a particular case. We investigate the behavior of the probability of the expert rule being optimal, as well as that of the majority rule, both as functions of the distribution parameter and the group size. The main result is that for any value of the distribution parameter the expert rule is far more likely to be optimal than the majority rule, especially as the deciding body becomes larger.
\end{abstract}

Keywords: Decision rule, Expert rule, Majority rule, Optimality, Probability

\section{Introduction}

In a wide variety of areas - science, business, family and others - decisions are to be made on a group basis. Even some nominally individual decisions are influenced by external advice and information. Thus group decision making is at least as important as individual decision making.

In this paper we focus on the binary choice model, which goes back at least as far as Condorcet [10]. In this model, a group of decision makers is required to select one of two alternatives, of which exactly one is regarded as correct. We assume the decision makers to be independent. Unlike the classical social choice model, the individuals in the group share a common goal - to identify the correct alternative. A decision rule is a rule for

$\dagger$ Requests for reprints should be sent to Daniel Berend, Departments of Mathematics and of Computer Science, Ben-Gurion University, Beer-Sheva, 84105, Israel.

* Research partially supported by an Eshkol Fellowship from the Israeli Science Ministry 
translating the individual opinions of the members into a group decision. If the size of the group is $n$, the number of all possible decision rules is $2^{2^{n}}$. A decision rule is optimal if it maximizes the probability that the group will make the correct decision for all possible combinations of opinions. If the probability of each member to make the right choice is known and the alternatives are symmetric, then the optimal decision rule is a weighted majority rule. (See Nitzan and Paroush [16], [17], [19].)

There are several directions in the study of the dichotomous choice model. Condorcet's Jury Theorem is studied in various setups. Some deal with the case where the correctness probabilities of the decision makers are not necessarily equal - Grofman, Owen and Feld [13], Miller [15], Young [22], Paroush [20], Berend and Paroush [2]. Others relate to the generalization whereby there may be some dependence between the group members Boland [8], Berg [4], [5] and Ladha [14]). Special attention has been drawn to special decision rules arising from indirect voting systems (see, for example, Boland, Proschan and Tong [9], Berg [6], Berg and Paroush [7]). In some of the models, the group has freedom in choosing the decision rule. The identification of the optimal rule is of primary importance here (see, for example, Nitzan and Paroush [16], [17], [19], Grofman et al [13], Gradstein and Nitzan [12]).

Our goal is identifying the optimal decision rule under partial information on the decision skills. Specifically, we assume the correctness probabilities of the group members to be independent random variables, distributed according to some given distribution rule. Moreover, while the values these variables take are unknown, we assume that the ranking of the members in terms of their individual correctness probabilities is (at least partly) known. Thus, one can follow rules based on this ranking. The extremes are the expert rule - following the advice of the most qualified individual while ignoring all the rest, and the majority rule - always taking the majority advice, even when advocated by mostly less qualified group members (strictly speaking, the latter rule is defined only for odd $n$ ). Clearly, there are numerous other decision rules in-between these two extremes.

Denote by $P_{\mathrm{e}}(n)$ the probability of the expert rule being optimal and by $P_{\mathrm{m}}(n)$ the probability of the majority rule being optimal. Nitzan and Paroush [19] obtained an explicit expression for $P_{\mathrm{e}}(n)$ for the case of log-normal distribution of the individual correctness probabilities. Nitzan and Paroush [18], [19] considered the case where these probabilities are uniformly distributed on $\left[\frac{1}{2}, 1\right]$. Using Monte Carlo method they estimated $P_{\mathrm{e}}(n)$ for small values of $n$, from 3 to 9 . They also identified all rules which could be optimal for $n=5$, and again by Monte Carlo estimated the probability of each of these being optimal. It turns out that the majority 
rule comes last, with $P_{\mathrm{m}}(5)=0.022$, while the expert rule is the second best, with $P_{\mathrm{e}}(5)=0.199$, and the leading one is the balanced expert rule of order 4 , given by the weights $(2,1,1,1,0)$, with probability 0.229 of being optimal. This line of research was continued by Berend and Harmse [1], still for uniform distribution on $\left[\frac{1}{2}, 1\right]$. They obtained an explicit formula for $P_{\mathrm{e}}(n)$ and an upper bound for $P_{\mathrm{m}}(n)$. The combination of these results implies that the latter probability decays to 0 much quicker than the former. This direction was followed by Sapir [21], dealing with the situation of logarithmic expertise levels distributed exponentially. In this case, the probabilities $P_{\mathrm{e}}(n)$ and $P_{\mathrm{m}}(n)$ (as well as that of the so-called balanced expert rule of order $n$ ) were calculated explicitly. A comparison of these probabilities shows that, again, the expert rule has a much better chance of being optimal than the majority rule.

This paper continues in the direction of Berend and Harmse [1]. The correctness probabilities are drawn from a family of distributions, containing the uniform distribution as a particular case. Our goal is to compare the asymptotic behavior of the probability of the expert rule being optimal with that of the majority rule. It turns out again that for sufficiently large $n$ the probability of the expert rule being optimal exceeds by far that of the majority rule. Thus the main question addressed in this paper is not what the probability of each decision rule is to provide the right decision. These probabilities may be assumed to be quite high, and in fact converge to 1 as the number of experts increases, as long as a "reasonable" decision rule is employed. These probabilities are what could be called "the average case". Here we deal rather with "the worst case". Namely, each decision rule has some borderline cases. One should hesitate to use the majority rule if, say, in a committee comprising of 11 members, the 6 members known to be least qualified happen to favor one view while all 5 more qualified members hold the opposite view. Similarly, employing the expert rule would seem strange if the top expert is opposed by all the others. To claim that, in a specific case, the majority rule (expert rule, respectively) is optimal, is tantamount to asserting that we should indeed favor the opinion of the 6 against the 5 in the first example (of the top expert in the second example, respectively). Consequently, comparing the optimality probabilities, as done in this paper, we cannot conclude that one rule is better than another. Rather, it provides us with a view of the performance of the rules in question in some extreme cases, and hints to what extent we should rather modify them in those cases.

Section 2 is devoted to a more accurate description of our model. In Section 3 we present the main results, and in Section 4 -their proofs. 
The authors express their gratitude to J. Rayner and to the referees for their very helpful comments on the first draft of the paper.

\section{The Model}

The group consists of $n$ members, faced by two alternatives, of which exactly one is correct. The alternatives are assumed to be symmetric. Namely, they are a priori equally likely to be correct, and the benefit (or loss) associated with a success (correct choice) or a failure (incorrect choice) is independent of the particular alternative correctly (or incorrectly) chosen. We assume that the members are independent in their choices. Denote by $p_{i}$ the probability of the $i$ th expert making the right choice. The vector $\vec{p}=\left(p_{1}, p_{2}, \ldots, p_{n}\right)$ is called the vector of abilities or skills.

Designating the alternatives (in an arbitrary way) as first and second, we also define the random variables

$$
X_{i}=\left\{\begin{aligned}
1, & \text { the } i \text { th expert selects the first alternative } \\
-1, & \text { the } i \text { th expert selects the second alternative. }
\end{aligned}\right.
$$

A decision profile $\vec{x}=\left(x_{1}, x_{2}, \ldots, x_{n}\right)$ is the $n$-tuple obtained from a specific instance of these variables. A decision rule is a rule for translating the individual opinions into a group decision. More formally, using the terminology of Nitzan and Paroush [19], a decision rule $\varphi$ is a function from the set of all possible decision profiles to the set $\{-1,1\}$. If $\varphi(\vec{x})=1$ then the first alternative is selected, while if $\varphi(\vec{x})=-1$ then the second alternative is selected. The number of all possible decision rules is $2^{2^{n}}$. A decision rule is neutral if $\varphi\left(-x_{1},-x_{2}, \ldots,-x_{n}\right)=-\varphi\left(x_{1}, x_{2}, \ldots, x_{n}\right)$ for every decision profile $\vec{x}=\left(x_{1}, x_{2}, \ldots, x_{n}\right)$. We assume our decision rules to be neutral. A decision rule is optimal if it maximizes the probability of the group to make the correct decision for all possible combinations of opinions. If the members indexed by some subset $A \subseteq\{1,2, \ldots, n\}$ of the group recommend the first alternative, while those indexed by $B=\{1, \ldots, n\} \backslash A$ recommend the second, then the first alternative should be chosen if and only if

$$
\prod_{i \in A} \frac{p_{i}}{1-p_{i}}>\prod_{i \in B} \frac{p_{i}}{1-p_{i}}
$$

or, equivalently,

$$
\sum_{i \in A} \ln \left(\frac{p_{i}}{1-p_{i}}\right)>\sum_{i \in B} \ln \left(\frac{p_{i}}{1-p_{i}}\right)
$$


(see Nitzan and Paroush [16], [17], [19]). The optimal decision rule for a group of $n$ experts turns out to be a weighted majority rule. Here a decision rule is a weighted majority rule if it satisfies $\varphi(\vec{x})=\operatorname{sign}\left(\sum_{i=1}^{n} w_{i} x_{i}\right)$ for every decision profile $\vec{x}$, where $\vec{w}=\left(w_{1}, w_{2}, \ldots, w_{n}\right)$ has non-negative entries and satisfies $\sum_{i=1}^{n} w_{i} x_{i} \neq 0$ for any profile $\vec{x}$. Such a vector $\vec{w}$ is a system of weights. Note that a weighted majority rule may be represented by many systems of weights.

In view of (2.1) and (2.2) it is natural to define the expertise of an individual, whose probability of being correct is $p$, as $\frac{p}{1-p}$, and his logarithmic expertise as $\ln \left(\frac{p}{1-p}\right)$. It will be convenient to consider the functions $F$ and $f$ defined by:

$$
F(p)=\frac{p}{1-p}, \quad f(p)=\ln \frac{p}{1-p}=\ln F(p) .
$$

With these notations, (2.2) may be restated to say that the optimal decision rule is a weighted majority rule, with weights $w_{i}=f\left(p_{i}\right)$. In other words, $\varphi(\vec{x})=\operatorname{sign}\left(\sum_{i=1}^{n} f\left(p_{i}\right) x_{i}\right)$ (see Nitzan and Paroush [19], Theorem 2.3.1).

In the sequel, we will employ multi-dimensional versions of the functions $f$ and $F$, denoted in the same way. Thus, the combined expertise of a set of $l$ experts with correctness probabilities $p_{1}, \ldots, p_{l}$ is $F\left(p_{1}, \ldots, p_{l}\right)=$ $\prod_{j=1}^{l} F\left(p_{j}\right)$, and the combined logarithmic expertise of the same experts is $f\left(p_{1}, \ldots, p_{l}\right)=\sum_{j=1}^{l} f\left(p_{j}\right)$.

\section{Main Results}

The results in this section attempt to generalize those of the case of uniform distribution, discussed by Berend and Harmse [1]. We are concerned with the probability $P_{\mathrm{e}}(n)$ of the expert rule being optimal and the probability $P_{\mathrm{m}}(n)$ for the majority rule being optimal. One verifies easily that $P_{\mathrm{e}}(n)$ is the probability that, in the case the top expert disagrees with all other experts, the top expert is more likely to be correct than the rest. Strictly speaking, $P_{\mathrm{m}}(n)$ is defined only for odd $n=2 s+1$. It is equal to the probability that the bottom $s+1$ experts, when opposed by the top $s$ experts, are more likely to be correct than the top ones. Our primary goal is to compare the probability that the expert rule is optimal with the probability that the majority rule is optimal. The point of this comparison is that it substantiates the conclusion obtained by Berend and Harmse [1] in the uniform case, by showing that the expert rule is far more likely to be optimal than the majority rule for large $n$, for a family of distributions, 
which contains the uniform distribution as a very particular case. Here we assume that the probability $p_{i}$ of the $i$-th expert to make a correct choice is distributed according to the (same) density function

$$
\rho(x)= \begin{cases}2 \alpha(2 x-1)^{\alpha-1}, & \frac{1}{2} \leq x \leq 1, \\ 0, & \text { otherwise }\end{cases}
$$

where $\alpha$ is any positive parameter. If $\alpha=1$ we get the uniform distribution $U\left[\frac{1}{2}, 1\right]$ as a special instance of $(3.1)$.

Why should one consider this family of distributions? The uniform distribution in the interval $\left[\frac{1}{2}, 1\right]$ looks like a natural guess when one has no information on the distribution, yet assumes that people perform better by considering the possibilities than by tossing a coin. Yet, the results obtained in the case of the uniform distribution may seem too narrow, and invite the question as to how applicable they are if the distribution is somewhat perturbed. The family selected here is wide enough to cover both cases where the experts are mostly correct (large $\alpha$ ) and where they mostly perform only marginally better than coin tossing $(\alpha \approx 0)$. Let us also mention in passing, that if $p_{i}$ is distributed as in (3.1), and we denote $q_{i}=1-p_{i}$, then $\frac{1}{p_{i}-q_{i}}$ is distributed Pareto with parameter $\alpha$. Thus the results of the current paper prove the robustness of those obtained earlier only for the uniform distribution.

It will be convenient to introduce the following constants:

$$
\mathcal{I}_{k}=2 \alpha \int_{0}^{1} \frac{(1-t)^{\alpha-1} t^{k}}{(1+t)^{\alpha+1}} d t, \quad k=0,1,2, \ldots .
$$

It is easy to see that $1=\mathcal{I}_{0}>\mathcal{I}_{1}>\mathcal{I}_{2}>\ldots$.

The following theorem describes the asymptotic behavior of $P_{\mathrm{e}}(n)$.

THEOREM 1 The probability of the expert rule being optimal decays exponentially as the number of experts grows. More precisely:

1) If $0<\alpha \leq \frac{1}{2}$, then

$$
2 \alpha n \mathcal{I}_{1}^{n-1}-2 \alpha^{2} n \mathcal{I}_{2}^{n-1} \leq P_{\mathrm{e}}(n) \leq 2 \alpha n \mathcal{I}_{1}^{n-1}-2 \alpha^{2} n \mathcal{I}_{2}^{n-1}+n \mathcal{I}_{3}^{n-1} .
$$

2) If $\alpha \geq \frac{1}{2}$, then

$$
2 \alpha n \mathcal{I}_{1}^{n-1}-2 \alpha^{2} n \mathcal{I}_{2}^{n-1} \leq P_{\mathrm{e}}(n) \leq 2 \alpha n \mathcal{I}_{1}^{n-1}
$$


Remark 1. Clearly, for large $n$ we may omit the last two terms on the right hand side of (3.2) and obtain the (less accurate but simpler) bound of (3.3). However, this is not the case for small $n$.

Our next result relates to $P_{\mathrm{m}}(n)$. It provides an upper bound for it.

THEOREM 2 For odd-size $n=2 s+1$, the probability of the majority rule being optimal is bounded above as follows:

1) If $0<\alpha \leq 1$, then

$$
P_{\mathrm{m}}(n) \leq \frac{\alpha^{s}}{s !^{2}}
$$

2) If $\alpha>1$, then

$$
P_{\mathrm{m}}(n) \leq \frac{n}{s !^{2}} \cdot\left(\frac{\alpha}{2}\right)^{s} .
$$

In Theorem 2 we chose the simplest form of upper bounds for $P_{\mathrm{m}}(n)$. Actually, our calculations enable us to obtain more accurate results, given by Theorem 3 below. To present them we use the following notations:

$$
\begin{aligned}
& A_{s, \alpha}=\int_{0}^{1}\left(\left(1-x^{2}\right) x^{2 \alpha-1} \ln \frac{1+x}{1-x}\right)^{s} x^{\alpha-1} d x, \\
& B_{s, \alpha}=\int_{0}^{1}\left(\left(1-x^{2}\right) x^{\alpha-1} \ln ^{2} \frac{1+x}{1-x}\right)^{s} x^{\alpha-1} d x .
\end{aligned}
$$

THEOREM 3 For odd-size $n=2 s+1$, the probability of the majority rule being optimal is bounded above as follows:

1) If $0<\alpha \leq 1$, then

$$
P_{\mathrm{m}}(n) \leq \frac{\alpha n}{s !^{2}}\left(\frac{\alpha}{2}\right)^{s} A_{s, \alpha} \leq \frac{n}{s !^{2}}\left(\frac{2}{3}\left(\frac{3 \alpha}{2 \alpha+2}\right)^{\alpha+1}\right)^{s} .
$$

2) If $\alpha>1$, then

$$
P_{\mathrm{m}}(n) \leq \frac{\alpha n}{s !^{2}}\left(\frac{\alpha^{2}}{2(\alpha+1)}\left(\frac{\alpha-1}{\alpha+1}\right)^{\frac{\alpha-1}{2}}\right)^{s} B_{s, \alpha} \leq \frac{n}{s !^{2}}\left(\frac{\ln (4 \alpha+7.14)}{e}\right)^{n-1} .
$$

Remark 2. For $\alpha=1$, Theorem 3 yields $P_{\mathrm{m}}(n) \leq \frac{n}{s !^{2}}\left(\frac{3}{8}\right)^{s}$, which is somewhat worse than the upper bound $P_{\mathrm{m}}(n) \leq \frac{n}{s !^{2}}\left(\frac{1}{3}\right)^{s}$ obtained by 
Berend and Harmse [1]. Our method enables us to obtain tighter bounds for $0<\alpha \leq 1$ by simply taking more terms of the relevant Taylor series in the proof. For example, taking one more term we get for $0<\alpha \leq 1$ :

$$
P_{\mathrm{m}}(n) \leq \frac{n}{s !^{2}}\left(\frac{\alpha^{\alpha+1}}{3^{1-\alpha}(2+\alpha)^{2}} \cdot \frac{11 \alpha+14-\sqrt{55(\alpha+1)^{2}-30}}{\left(\alpha+1+\frac{1}{5} \sqrt{55(\alpha+1)^{2}-30}\right)^{\alpha}}\right)^{s}
$$

For $\alpha=1$ this already implies $P_{\mathrm{m}}(n) \leq \frac{n}{s !^{2}}\left(\frac{1}{3}\right)^{s}$. The derivation of (3.5) will be explained after the proof of Theorem 2 .

Combining the above theorems, we can compare the asymptotic behavior of $P_{\mathrm{e}}(n)$ and $P_{\mathrm{m}}(n)$. We see that, for any value of $\alpha, P_{\mathrm{e}}(n)$ decays to 0 exponentially as $n$ increases, while $P_{\mathrm{m}}(n)$ decays to 0 super-exponentially fast.

Theorem 1 also shows the influence of $\alpha$ on the probability of the expert rule being optimal. In fact, routine estimates show that

$$
\frac{1}{2(\alpha+1)} \leq \mathcal{I}_{1} \leq \frac{1}{\alpha+1} .
$$

This shows that, as a function of $\alpha, P_{\mathrm{e}}(n)$ decreases as $\frac{1}{\alpha^{n-2}}$ for large $\alpha$. Table 1 provides the values of $P_{\mathrm{e}}(n)$ for a few values of $\alpha$ and $n$. The calculations were done by Monte Carlo method using $10^{4}$ iterations.

Table 1. The probability of the expert rule being optimal.

\begin{tabular}{|l|ccccccccc|}
\hline$\alpha$ & 0.05 & 0.25 & 0.5 & 1 & 1.5 & 2 & 3 & 10 & 100 \\
& & & & & & & & & \\
\hline 3 & 0.99 & 0.94 & 0.85 & 0.68 & 0.57 & 0.48 & 0.36 & 0.14 & 0.02 \\
5 & 0.98 & 0.74 & 0.48 & 0.20 & 0.10 & 0.05 & 0.02 & 0.00 & 0.00 \\
7 & 0.95 & 0.54 & 0.23 & 0.05 & 0.01 & 0.00 & 0.00 & 0.00 & 0.00 \\
15 & 0.79 & 0.10 & 0.01 & 0.00 & 0.00 & 0.00 & 0.00 & 0.00 & 0.00 \\
\hline
\end{tabular}

Numerical calculations tend to show that the influence of $\alpha$ on $P_{\mathrm{m}}(n)$ is opposite to its influence on $P_{\mathrm{e}}(n)$. Thus Table 2 indicates that, as a function of $\alpha, P_{\mathrm{m}}(n)$ is increasing. (Note that this also agrees with what one might guess in view of Theorem 2.) The data was again computed by Monte Carlo method.

Figure 1 represents graphically the data of Table 1 and 2 in the case $n=5$. 
Table 2. The probability of the majority rule being optimal.

\begin{tabular}{|l|ccccccccc|}
\hline$\alpha$ & 0.05 & 0.25 & 0.5 & 1 & 1.5 & 2 & 3 & 10 & 100 \\
& & & & & & & & & \\
\hline 3 & 0.01 & 0.06 & 0.15 & 0.32 & 0.43 & 0.52 & 0.64 & 0.86 & 0.99 \\
5 & 0.00 & 0.00 & 0.00 & 0.02 & 0.05 & 0.07 & 0.13 & 0.40 & 0.85 \\
7 & 0.00 & 0.00 & 0.00 & 0.00 & 0.00 & 0.00 & 0.01 & 0.08 & 0.49 \\
15 & 0.00 & 0.00 & 0.00 & 0.00 & 0.00 & 0.00 & 0.00 & 0.00 & 0.00 \\
\hline
\end{tabular}

Figure 1. $P_{\mathrm{e}}(5)$ and $P_{\mathrm{m}}(5)$ as functions of $\alpha$

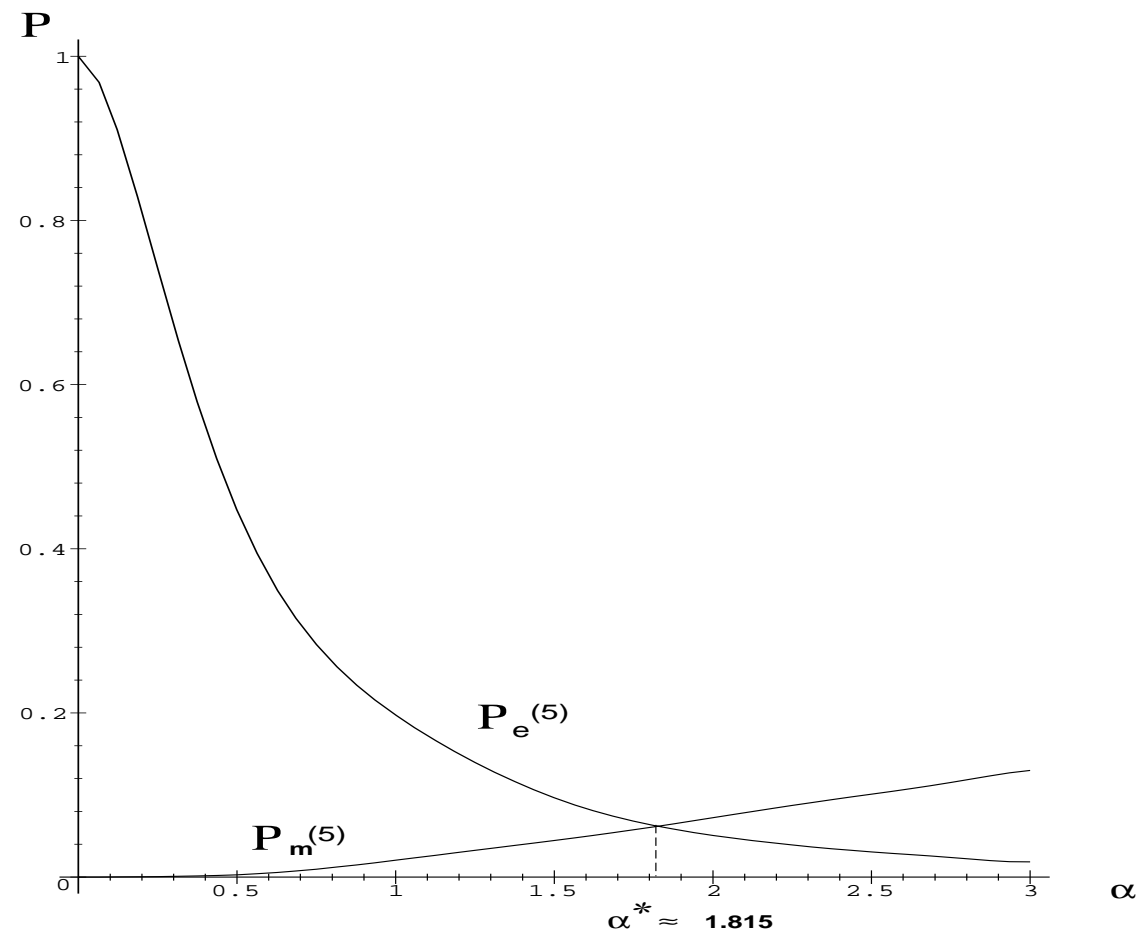


For integer $\alpha=m, \rho(x)$ is a polynomial on $\left[\frac{1}{2}, 1\right]$. In this case, one can obtain an exact expression for $P_{\mathrm{e}}(n)$ in terms of an infinite Leibniz-type series. An expansion of this type may be obtained for $\alpha=\frac{1}{2}$ as well. We refer to Berend and Sapir [3] for details.

\section{Proofs}

For the proof of Theorem 1 we need the following lemma.

LEMMA 1 The following inequalities hold:
1) $(1-x)^{\beta} \geq 1-\beta x$, $0 \leq x \leq 1, \quad \beta \geq 1$
2) $\left(\frac{1-x}{1+x}\right)^{\beta} \geq 1-2 \beta x+2 \beta^{2} x^{2}-x^{3}, 0 \leq x \leq 1,0<\beta \leq \frac{1}{2}$.
3) $\left(\frac{1-x}{1+x}\right)^{\beta} \leq 1-2 \beta x+2 \beta^{2} x^{2}, \quad 0 \leq x \leq 1, \beta>0$.

The proof is technical, and we omit the details.

Proof of Theorem 1: Let $p, q_{1}, \ldots, q_{n-1}$ be independent random variables with the same density $\rho(x)$. Denote $\vec{q}=\left(q_{1}, \ldots, q_{n-1}\right), \quad F(\vec{q})=$ $\prod_{i=1}^{n-1} F\left(q_{i}\right)$ and $d \vec{q}=d q_{1} \ldots d q_{n-1}$. Note that $F^{-1}(t)=\frac{t}{1+t}$. Then:

$$
\begin{aligned}
& P_{\mathrm{e}}(n)=n \cdot \operatorname{Prob}\left(F(p)>\prod_{i=1}^{n-1} F\left(q_{i}\right)\right) \\
& =n(2 \alpha)^{n} \underbrace{\int \ldots \int}_{\left[\frac{1}{2}, 1\right]^{n-1}} \prod_{i=1}^{n-1}\left(2 q_{i}-1\right)^{\alpha-1} \int_{F^{-1}(F(\vec{q}))}^{1}(2 p-1)^{\alpha-1} d p d \vec{q} \\
& =n(2 \alpha)^{n-1} \underbrace{\int \ldots \int}_{\left[\frac{1}{2}, 1\right]^{n-1}} \prod_{i=1}^{n-1}\left(2 q_{i}-1\right)^{\alpha-1}\left(1-\left(\frac{2 F(\vec{q})}{1+F(\vec{q})}-1\right)^{\alpha}\right) d \vec{q} \\
& =n-n(2 \alpha)^{n-1} \underbrace{\int \ldots \int}_{\left[\frac{1}{2}, 1\right]^{n-1}} \prod_{i=1}^{n-1}\left(2 q_{i}-1\right)^{\alpha-1}\left(\frac{2 F(\vec{q})}{1+F(\vec{q})}-1\right)^{\alpha} d \vec{q}
\end{aligned}
$$


Substituting $t_{i}=\frac{1-q_{i}}{q_{i}}$ and using the inequality $\frac{1}{1+\prod_{i=1}^{n-1} t_{i}} \geq 1-\prod_{i=1}^{n-1} t_{i}$, we obtain:

$$
\begin{aligned}
P_{\mathrm{e}}(n) & =n-n(2 \alpha)^{n-1} \underbrace{\int \ldots \int}_{[0,1]^{n-1}}\left(\prod_{i=1}^{n-1} \frac{\left(1-t_{i}\right)^{\alpha-1}}{\left(1+t_{i}\right)^{\alpha+1}}\right)\left(\frac{1-\prod_{i=1}^{n-1} t_{i}}{1+\prod_{i=1}^{n-1} t_{i}}\right)^{\alpha} d \vec{t} \\
& \leq n-n(2 \alpha)^{n-1} \underbrace{\int \ldots \int}_{[0,1]^{n-1}}\left(\prod_{i=1}^{n-1} \frac{\left(1-t_{i}\right)^{\alpha-1}}{\left(1+t_{i}\right)^{\alpha+1}}\right)\left(1-\prod_{i=1}^{n-1} t_{i}\right)^{2 \alpha} d \vec{t} .
\end{aligned}
$$

If $\alpha \geq \frac{1}{2}$, then by Lemma 1.1 with $x=\prod_{i=1}^{n-1} t_{i}$ and $\beta=2 \alpha$ this implies

$$
P_{\mathrm{e}}(n) \leq n\left(1-\mathcal{I}_{0}^{n-1}+2 \alpha \mathcal{I}_{1}^{n-1}\right) .
$$

Now $\mathcal{I}_{0}=1$, and therefore

$$
P_{\mathrm{e}}(n) \leq 2 \alpha n \mathcal{I}_{1}^{n-1}
$$

Denote $x=\prod_{i=1}^{n-1} t_{i}$. Let $\alpha \leq \frac{1}{2}$. By Lemma 1.2 and the equality part of (4.1) we obtain the upper bound:

$$
\begin{aligned}
P_{\mathrm{e}}(n) & \leq n\left(1-\mathcal{I}_{0}^{n-1}+2 \alpha \mathcal{I}_{1}^{n-1}-2 \alpha^{2} \mathcal{I}_{2}^{n-1}+\mathcal{I}_{3}^{n-1}\right) \\
& =2 \alpha n \mathcal{I}_{1}^{n-1}-2 \alpha^{2} n \mathcal{I}_{2}^{n-1}+n \mathcal{I}_{3}^{n-1}
\end{aligned}
$$

The equality part of (4.1) and Lemma 1.3 provide a lower bound for $P_{\mathrm{e}}(n)$ :

$$
\begin{aligned}
P_{\mathrm{e}}(n) & \geq n\left(1-\mathcal{I}_{0}^{n-1}+2 \alpha \mathcal{I}_{1}^{n-1}-2 \alpha^{2} \mathcal{I}_{2}^{n-1}\right) \\
& =2 \alpha n \mathcal{I}_{1}^{n-1}-2 \alpha^{2} n \mathcal{I}_{2}^{n-1} .
\end{aligned}
$$

Combining (4.2), (4.3) and (4.4) we obtain the theorem.

The proof of Theorem 2 is a variation of part of the proof of Theorem 3 . It will therefore be convenient to prove the latter first.

Proof of Theorem 3: The proof will be carried out in three stages. In the first stage we transform our situation to one with a uniform distribution. In the second stage we obtain the left upper bounds for $P_{\mathrm{m}}(n)$ in the theorem, which contain the integrals $A_{s, \alpha}$ and $B_{s, \alpha}$. We will need to distinguish between two cases: $\alpha \leq 1$ and $\alpha>1$. The proof in each of these cases will be similar to that given by Berend and Harmse [1] in the special case 
$\alpha=1$. The third stage is technical, dealing with estimations of $A_{s, \alpha}$ and $B_{s, \alpha}$. Here we produce the final upper bounds for $P_{\mathrm{m}}(n)$.

Stage 1: Suppose the correctness probability of the middle (i.e., $(s+$ 1)st) expert is $a$ for some $a \in\left[\frac{1}{2}, 1\right]$. Denote the conditional correctness probabilities of the $s$ top experts by $P_{1}, P_{2}, \ldots, P_{s}$, and those of the $s$ bottom experts by $Q_{1}, Q_{2}, \ldots, Q_{s}$. All variables are independent, with the first $s$ taking values in $[a, 1]$, and the latter $s-$ in $\left[\frac{1}{2}, a\right]$. With these notations, the desired probability is the probability that the inequality

$$
f\left(Q_{1}, Q_{2}, \ldots, Q_{s}, a\right) \geq f\left(P_{1}, P_{2}, \ldots, P_{s}\right)
$$

holds. Denote $t_{i}=\frac{\left(2 p_{i}-1\right)^{\alpha}+1}{2}, \quad i=1,2, \ldots, 2 s+1$. It is easy to see that the random variables $t_{i}$ are i.i.d uniformly distributed in $\left[\frac{1}{2}, 1\right]$. Putting $\mu=\frac{(2 a-1)^{\alpha}+1}{2}$, and

$$
T_{j}=\frac{\left(2 P_{i}-1\right)^{\alpha}+1}{2}, \quad V_{j}=\frac{\left(2 Q_{i}-1\right)^{\alpha}+1}{2}, \quad j=1,2, \ldots, s,
$$

we observe that $T_{j} \sim \mathrm{U}[\mu, 1]$ and $V_{j} \sim \mathrm{U}[1 / 2, \mu]$ for each $j$, and all these variables are independent. Let $g(t)=\ln \frac{1+(2 t-1)^{1 / \alpha}}{1-(2 t-1)^{1 / \alpha}}$ be the logarithmic expertise, expressed as a function of $t$ (which corresponds to the variable $t_{i}$ ). As with the logarithmic expertise function $f$, we have a multi-dimensional version of $g$, also denoted by $g$, defined by

$$
g\left(t_{1}, t_{2}, \ldots, t_{k}\right)=\sum_{j=1}^{k} g\left(t_{i}\right) .
$$

Then (4.5) takes place if and only if:

$$
g\left(V_{1}, V_{2}, \ldots, V_{s}, \mu\right) \geq g\left(T_{1}, T_{2}, \ldots, T_{s}\right) .
$$

We shall use the first two derivatives of $g(t)$ :

$$
\begin{gathered}
g^{\prime}(t)=\frac{4}{\alpha} \cdot \frac{(2 t-1)^{\frac{1}{\alpha}-1}}{1-(2 t-1)^{2 / \alpha}}, \\
g^{\prime \prime}(t)=\frac{8}{\alpha^{2}} \cdot \frac{1-\alpha+(1+\alpha)(2 t-1)^{2 / \alpha}}{\left(1-(2 t-1)^{2 / \alpha}\right)^{2}(2 t-1)^{2-\frac{1}{\alpha}}} .
\end{gathered}
$$

Clearly, $g^{\prime}(t) \geq 0$ for $1 / 2 \leq t \leq 1$. With respect to $g^{\prime \prime}$, if $0<\alpha \leq 1$, then $g^{\prime \prime}$ is non-negative throughout the interval, whereas for $\alpha>1$ it changes 
its sign in the interval. Hence we have to distinguish between the cases $0<\alpha \leq 1$ and $\alpha>1$.

\section{Stage 2:}

Case 2.1: $0<\alpha<1$.

Since the one-variable form of $g$ is convex on $\left[\frac{1}{2}, 1\right]$ and $g\left(\frac{1}{2}\right)=0$, we obtain the upper bound:

$$
g\left(V_{j}\right) \leq \frac{V_{j}-\frac{1}{2}}{\mu-\frac{1}{2}} g(\mu)=g(\mu)-\frac{\mu-V_{j}}{\mu-\frac{1}{2}} g(\mu) .
$$

The convexity of $g$ also implies the lower bound:

$$
g\left(T_{j}\right) \geq g(\mu)+\left(T_{j}-\mu\right) g^{\prime}(\mu)=g(\mu)+\frac{T_{j}-\mu}{1-\mu} g^{\prime}(\mu)(1-\mu) .
$$

Thus the desired event (4.6) cannot hold unless

$$
g^{\prime}(\mu)(1-\mu) \sum_{j=1}^{s} X_{j}+g(\mu) \sum_{j=1}^{s} Y_{j} \leq g(\mu)
$$

where $X_{j}=\frac{T_{j}-\mu}{1-\mu}$ and $Y_{j}=\frac{\mu-V_{j}}{\mu-\frac{1}{2}}$ are independent uniformly distributed in $[0,1]$. As in the proof of Theorem 4 in Berend and Harmse [1], this implies that the probability of the event in (4.11) does not exceed

$$
\frac{g(\mu)^{n-1}}{\left(g(\mu) g^{\prime}(\mu)(1-\mu)\right)^{s}(n-1) !} .
$$

Now the correctness probability of the $(s+1)$ st expert is itself a random variable with density function

$$
\psi(\mu)= \begin{cases}2^{n}\left(\begin{array}{c}
n \\
s, s, 1
\end{array}\right)\left(\mu-\frac{1}{2}\right)^{s}(1-\mu)^{s}, & \frac{1}{2} \leq \mu \leq 1 \\
0, & \text { otherwise }\end{cases}
$$

(cf. Golberg [11], Sec. 11.5). Hence

$$
\begin{aligned}
P_{\mathrm{m}}(n) & =\int_{\frac{1}{2}}^{1} P\left(g\left(V_{1}, V_{2}, \ldots, V_{s}, \mu\right) \geq g\left(T_{1}, T_{2}, \ldots, T_{s}\right)\right) \psi(\mu) d \mu \\
& \leq \frac{1}{(n-1) !} \int_{\frac{1}{2}}^{1}\left(\frac{g(\mu)}{g^{\prime}(\mu)(1-\mu)}\right)^{s} \psi(\mu) d \mu .
\end{aligned}
$$


Substituting (4.7) and (4.12) in the last inequality, we obtain:

$$
\begin{aligned}
P_{\mathrm{m}}(n) \leq & \left(\frac{\alpha}{2}\right)^{s} \frac{2 n}{s !^{2}} \\
& \cdot \int_{\frac{1}{2}}^{1}\left(1-(2 \mu-1)^{\frac{2}{\alpha}}\right)^{s}(2 \mu-1)^{s\left(2-\frac{1}{\alpha}\right)}\left(\ln \frac{1+(2 \mu-1)^{1 / \alpha}}{1-(2 \mu-1)^{1 / \alpha}}\right)^{s} d \mu .
\end{aligned}
$$

The change of variable $x=(2 \mu-1)^{1 / \alpha}$ yields the first inequality asserted in this case:

$$
\begin{aligned}
P_{\mathrm{m}}(n) & \leq\left(\frac{\alpha}{2}\right)^{s+1} \frac{2 n}{s !^{2}} \int_{0}^{1}\left(\left(1-x^{2}\right) x^{2 \alpha-1} \ln \frac{1+x}{1-x}\right)^{s} x^{\alpha-1} d x \\
& =\frac{\alpha n}{s !^{2}}\left(\frac{\alpha}{2}\right)^{s} A_{s, \alpha},
\end{aligned}
$$

Case 2.2: $\alpha>1$.

By (4.8) we see that $g^{\prime \prime}(t)$ changes signs in the interval $\left[\frac{1}{2}, 1\right]$. In fact, $g^{\prime \prime}$ vanishes at the point $t_{\alpha}=\frac{1}{2}+\frac{1}{2}\left(\frac{\alpha-1}{\alpha+1}\right)^{\alpha / 2}$, so that $g^{\prime \prime}(t)<0$ for $t \in\left[\frac{1}{2}, t_{\alpha}\right)$ and $g^{\prime \prime}(t)>0$ for $t \in\left(t_{\alpha}, 1\right]$. Thus in this case $g(t)$ is concave in $\left[\frac{1}{2}, t_{\alpha}\right)$ and convex in $\left(t_{\alpha}, 1\right]$. We proceed similarly to Case 2.1. When estimating the integrand in

$$
\int_{\frac{1}{2}}^{1} P\left(g\left(V_{1}, V_{2}, \ldots, V_{s}, \mu\right) \geq g\left(T_{1}, T_{2}, \ldots, T_{s}\right)\right) \psi(\mu) d \mu
$$

as a function of $\mu$, we shall proceed differently for $\mu>t_{\alpha}$ and for $\mu<t_{\alpha}$. Namely, write:

$$
\begin{aligned}
P_{\mathrm{m}}(n) & =\int_{\frac{1}{2}}^{1} P\left(g\left(V_{1}, V_{2}, \ldots, V_{s}, \mu\right) \geq g\left(T_{1}, T_{2}, \ldots, T_{s}\right)\right) \psi(\mu) d \mu \\
& =\int_{\frac{1}{2}}^{t_{\alpha}}+\int_{t_{\alpha}}^{1} .
\end{aligned}
$$

We first consider the integral over the region $\left[t_{\alpha}, 1\right]$. Since $g(t)$ is convex on $[\mu, 1]$, the lower bound (4.10) on $g\left(T_{j}\right)$ is still valid. Bounding the $g\left(V_{j}\right)$ 's from above is less obvious. We want to bound $g(t)$ on interval $\left[\mu, \frac{1}{2}\right]$ by a linear function. To this end, we use the unique point $t_{0} \in\left[\frac{1}{2}, t_{\alpha}\right]$ such that the tangent to the graph of $g$ at the point $t_{0}$ passes through $(\mu, g(\mu))$. This tangent provides a linear function as required, and in particular:

$$
g\left(V_{j}\right) \leq g(\mu)-g^{\prime}\left(t_{0}\right)\left(\mu-V_{j}\right)=g(\mu)-g^{\prime}\left(t_{0}\right)\left(\mu-\frac{1}{2}\right) \frac{\mu-V_{j}}{\mu-\frac{1}{2}} .
$$

The following figure shows the tangents used in (4.10) and (4.15). 
Figure 2. Linear bounds for $g$ when $\mu>t_{\alpha}$

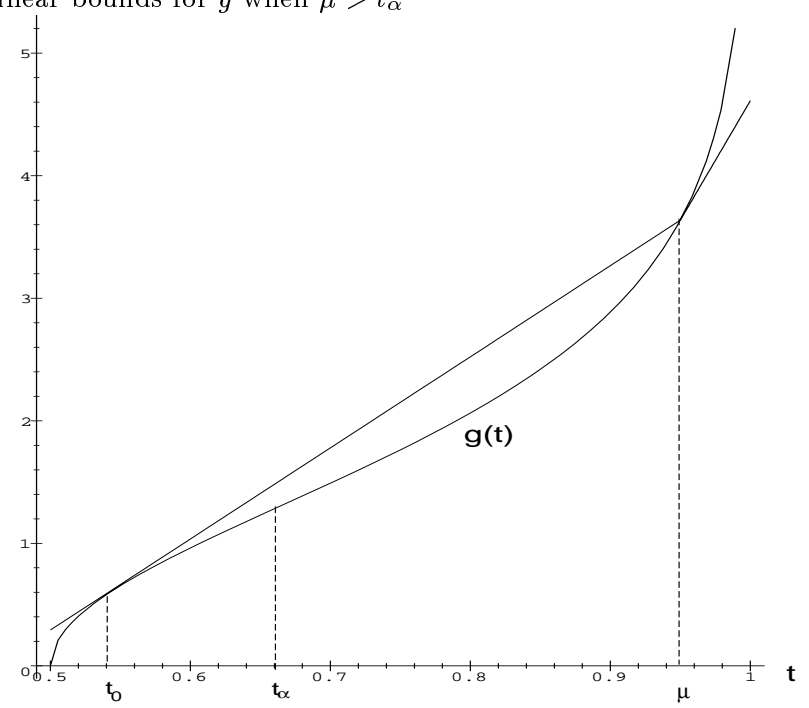

Thus (4.10) and (4.15) imply that (4.6) cannot hold unless

$$
g^{\prime}(\mu)(1-\mu) \sum_{j=1}^{s} X_{j}+g^{\prime}\left(t_{0}\right)\left(\mu-\frac{1}{2}\right) \sum_{j=1}^{s} Y_{j} \leq g(\mu),
$$

where $X_{j}=\frac{T_{j}-\mu}{1-\mu}$ and $Y_{j}=\frac{\mu-V_{j}}{\mu-\frac{1}{2}}$. Denote the probability of the event (4.16) by $\operatorname{Prob}_{1}(n)$. As in Case 2.1,

$$
\operatorname{Prob}_{1}(n) \leq \frac{g(\mu)^{n-1}}{(n-1) !\left(g^{\prime}\left(t_{0}\right)\left(\mu-\frac{1}{2}\right) g^{\prime}(\mu)(1-\mu)\right)^{s}} .
$$

Since $g$ is concave in $\left[\frac{1}{2}, t_{\alpha}\right]$, we have $g^{\prime}\left(t_{\alpha}\right) \leq g^{\prime}\left(t_{0}\right)$. Hence:

$$
\operatorname{Prob}_{1}(n) \leq \frac{g(\mu)^{n-1}}{(n-1) !\left(g^{\prime}\left(t_{\alpha}\right)\left(\mu-\frac{1}{2}\right) g^{\prime}(\mu)(1-\mu)\right)^{s}} .
$$

Now consider the first integral on the right hand side of (4.14). The function $g(t)$ is concave for $t \in\left[\frac{1}{2}, \mu\right]$, and (4.9) takes place. We want to bound $g$ on $(\mu, 1]$ from below by a linear function. Let $t_{0}^{*} \in\left(t_{\alpha}, 1\right)$ be the unique point such that the tangent to the graph of $g$ at the point $t_{0}^{*}$ passes 
through $(\mu, g(\mu))$. This tangent lies below the graph of $g$ throughout $[\mu, 1]$, that is:

$$
g\left(T_{j}\right) \geq g(\mu)+g^{\prime}\left(t_{0}^{*}\right)\left(T_{j}-\mu\right)=g(\mu)+g^{\prime}\left(t_{0}^{*}\right)(1-\mu) \frac{T_{j}-\mu}{1-\mu} .
$$

The following figure explains pictorially (4.9) and (4.18).

Figure 3. Linear bounds for $g$ when $\mu<t_{\alpha}$

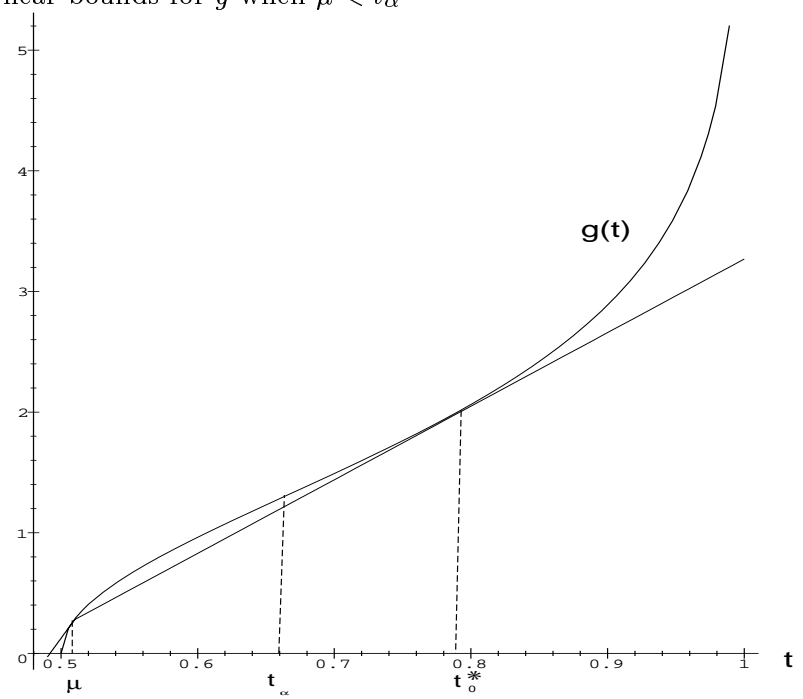

Thus (4.9) and (4.18) imply that (4.6) cannot hold unless

$$
g^{\prime}\left(t_{0}^{*}\right)(1-\mu) \sum_{j=1}^{s} X_{j}+g^{\prime}(\mu)\left(\mu-\frac{1}{2}\right) \sum_{j=1}^{s} Y_{j} \leq g(\mu),
$$

where $X_{j}$ and $Y_{j}$ are as before. Denote the probability of the event in (4.19) by $\operatorname{Prob}_{2}(n)$. Again, similarly to Case 2.1 we obtain

$$
\operatorname{Prob}_{2}(n) \leq \frac{g(\mu)^{n-1}}{(n-1) !\left(g^{\prime}\left(t_{0}^{*}\right)\left(\mu-\frac{1}{2}\right) g^{\prime}(\mu)(1-\mu)\right)^{s}} .
$$

Since $g$ is convex in $\left[t_{\alpha}, 1\right]$, we have $g^{\prime}\left(t_{\alpha}\right) \leq g^{\prime}\left(t_{0}^{*}\right)$. Hence:

$$
\operatorname{Prob}_{2}(n) \leq \frac{g(\mu)^{n-1}}{(n-1) !\left(g^{\prime}\left(t_{\alpha}\right)\left(\mu-\frac{1}{2}\right) g^{\prime}(\mu)(1-\mu)\right)^{s}} .
$$


Fortunately, the right hand sides of (4.17) and of (4.20) are the same. Substituting (4.17) and (4.20) in (4.14) we obtain:

$$
P_{\mathrm{m}}(n) \leq \int_{\frac{1}{2}}^{1} \frac{g(\mu)^{2 s}}{(n-1) !\left(g^{\prime}\left(t_{\alpha}\right)\left(\mu-\frac{1}{2}\right) g^{\prime}(\mu)(1-\mu)\right)^{s}} \psi(\mu) d \mu .
$$

By (4.12):

$$
P_{\mathrm{m}}(n) \leq 2^{n}\left(\begin{array}{c}
n \\
s, s, 1
\end{array}\right) \frac{1}{(n-1) !\left(g^{\prime}\left(t_{\alpha}\right)\right)^{s}} \int_{0}^{1} \frac{g(\mu)^{2 s}}{\left(g^{\prime}(\mu)\right)^{s}} d \mu .
$$

Replacing $g(\mu)$ and $g^{\prime}(\mu)$ by their explicit values and substituting $x=$ $(2 \mu-1)^{1 / \alpha}$, we get:

$$
P_{\mathrm{m}}(n) \leq \frac{\alpha n}{s !^{2}}\left(\frac{\alpha}{g^{\prime}\left(t_{\alpha}\right)}\right)^{s} \int_{0}^{1}\left(\left(1-x^{2}\right) x^{\alpha-1} \ln ^{2} \frac{1+x}{1-x}\right)^{s} x^{\alpha-1} d x .
$$

Plugging in the value of $g^{\prime}\left(t_{\alpha}\right)$ we arrive at the first inequality for part 2.

Stage 3: For the second inequality in part 1, we have to bound $A_{s, \alpha}$ from above. Denote:

$$
h(x)=\left(1-x^{2}\right) x^{2 \alpha-1} \ln \frac{1+x}{1-x}, \quad x \in[0,1] .
$$

We claim that

$$
h(x) \leq h_{1}(x)=2 x^{2 \alpha}\left(1-\frac{2}{3} x^{2}\right), \quad x \in[0,1] .
$$

Indeed, using Taylor's expansion of $\ln \frac{1+x}{1-x}$ we obtain:

$$
h(x)=\left(1-x^{2}\right) x^{2 \alpha-1} \cdot 2 x \sum_{k=0}^{\infty} \frac{x^{2 k}}{2 k+1}=2 x^{2 \alpha}\left(1-2 \sum_{k=1}^{\infty} \frac{x^{2 k}}{4 k^{2}-1}\right) .
$$

Taking only the first term in the sum on the right hand side of (4.23), we obtain (4.22). Since $h_{1}(x)$ attains its maximum at the point $x^{*}=\sqrt{\frac{3 \alpha}{2 \alpha+2}}$, we have

$$
h(x) \leq h_{1}\left(x^{*}\right)=\frac{2}{\alpha+1}\left(\frac{3 \alpha}{2(\alpha+1)}\right)^{\alpha}, \quad x \in[0,1] .
$$

Substituting in the expression (3.4) for $A_{s, \alpha}$ and combining with (4.13), we arrive at the second inequality in part 1 . 
To complete the proof of part 2, we need to estimate $B_{s, \alpha}$. Denote:

$$
\begin{aligned}
& \phi(x)=\ln \frac{1+x}{1-x}, \\
& h_{2}(x)=\left(1-x^{2}\right) x^{\alpha-1}, \\
& h_{3}(x)=h_{2}(x) \cdot \phi^{2}(x) .
\end{aligned}
$$

The better part of the remaining work is designed to find a good upper bound on $h_{3}(x)$ for $\alpha>1$ (see (4.29) infra). Clearly, $h_{3}(0)=h_{3}(1-)=0$ and $h_{3}(x)>0$ for $x \in(0,1)$. Let $x_{*} \in(0,1)$ be a maximum point of $h_{3}$. Putting $\psi(x)=4 x+\left(\alpha-1-(\alpha+1) x^{2}\right) \phi(x)$, we find that

$$
h_{3}^{\prime}(x)=x^{\alpha-2} \phi(x) \psi(x) .
$$

Since $h_{3}^{\prime}$ vanishes at $x_{*}$, so does $\psi$, and therefore

$$
\phi\left(x_{*}\right)=\frac{4 x_{*}}{(\alpha+1) x_{*}^{2}-(\alpha-1)} .
$$

The function $\frac{4 x}{(\alpha+1) x^{2}-(\alpha-1)}$ is negative in $\left(0, \sqrt{\frac{\alpha-1}{\alpha+1}}\right)$, and positive and decreasing in $\left(\sqrt{\frac{\alpha-1}{\alpha+1}}, 1\right)$. Since $\phi(x)$ is positive and increasing in $(0,1)$, there exists a unique point $x_{*}$ satisfying (4.25). Thus $x_{*}$ is the only maximum point of $h_{3}(x)$. A routine calculation shows that $\psi\left(\sqrt{\frac{\alpha+1.285}{\alpha+2.285}}\right)<0$ and $\psi\left(\frac{\alpha+4}{\alpha+5}\right)>0$ for $\alpha>1$, so that $x_{*} \in\left(\frac{\alpha+4}{\alpha+5}, \sqrt{\frac{\alpha+1.285}{\alpha+2.285}}\right)$.

Since $\phi(x)$ is increasing, for $x \in\left(\frac{\alpha+4}{\alpha+5}, \sqrt{\frac{\alpha+1.285}{\alpha+2.285}}\right)$ we have:

$$
\phi(x) \leq \phi\left(\sqrt{\frac{\alpha+1.285}{\alpha+2.285}}\right)=2 \ln (\sqrt{\alpha+2.285}+\sqrt{\alpha+1.285})
$$

The function $h_{2}$ decreases from the point $\sqrt{\frac{\alpha-1}{\alpha+1}}$ on, and in particular:

$$
h_{2}(x) \leq h_{2}\left(\frac{\alpha+4}{\alpha+5}\right), \quad x \in\left(\frac{\alpha+4}{\alpha+5}, \sqrt{\frac{\alpha+1.285}{\alpha+2.285}}\right) .
$$

Thus

$$
h_{3}(x) \leq h_{3}\left(x_{*}\right) \leq h_{2}\left(\frac{\alpha+4}{\alpha+5}\right) \phi^{2}\left(\sqrt{\frac{\alpha+1.285}{\alpha+2.285}}\right) .
$$

Combining (4.26), (4.27) and (4.28) we obtain:

$$
h_{3}\left(x_{*}\right) \leq \frac{2 \alpha+9}{(\alpha+5)^{2}}\left(\frac{\alpha+4}{\alpha+5}\right)^{\alpha-1} 4 \ln ^{2}(\sqrt{\alpha+2.285}+\sqrt{\alpha+1.285}) .
$$


Substituting (4.29) in the expression (3.4) for $B_{s, \alpha}$, we obtain:

$$
\begin{aligned}
P_{\mathrm{m}}(n) \leq \frac{n}{s !^{2}} & \left(\left(\frac{\alpha+4}{\alpha+5} \sqrt{\frac{\alpha-1}{\alpha+1}}\right)^{\alpha-1}\right. \\
& \left.\cdot \frac{4 \alpha^{2}(\alpha+4.5)}{(\alpha+5)^{2}(\alpha+1)} \ln ^{2}(\sqrt{\alpha+2.285}+\sqrt{\alpha+1.285})\right)^{s} .
\end{aligned}
$$

A lengthy but routine calculation shows that the right hand side in the last inequality is smaller than that in part 2 of the theorem, which concludes the proof.

Proof of Theorem 2: Denote $h_{4}(x)=\frac{1-x^{2}}{x} \ln \frac{1+x}{1-x}$ for $x \in[0,1]$. With $h(x)$ as in (4.21) we have $h_{4}(x)=h(x) / x^{2 \alpha}$, and therefore, by (4.23):

$$
h_{4}(x) \leq 2, \quad x \in[0,1] .
$$

Substituting this bound in the expression for $A_{s, \alpha}$, we arrive at:

$$
A_{s, \alpha} \leq 2^{s} \int_{0}^{1} x^{\alpha n-1} d x=2^{s} \frac{1}{\alpha n} .
$$

Combining this with (4.13), we complete the result in the first part of the theorem:

$$
P_{\mathrm{m}}(n) \leq \frac{\alpha^{s}}{s !^{2}}
$$

To complete the second part, denote:

$$
h_{5}(\alpha)=\frac{\alpha}{\alpha+1}\left(\frac{\alpha-1}{\alpha+1}\right)^{\frac{\alpha-1}{2}}, \quad h_{6}(\alpha)=\left(\frac{\alpha}{\alpha+1}\right)^{\frac{\alpha+1}{2}} .
$$

Clearly $h_{5}(\alpha) \leq h_{6}(\alpha)$. It is easy to see that $h_{5}(\alpha)$ is decreasing. A routine calculation reveals that the function $h_{7}(x)$, defined by

$$
h_{7}(x)=\left(1-x^{2}\right) \ln ^{2} \frac{1+x}{1-x}, \quad x \in[0,1),
$$

is bounded above by 1.76 , which produces:

$$
P_{\mathrm{m}}(n) \leq \frac{n}{s !^{2}\left(s\left(1-\frac{1}{\alpha}\right)+1\right)}\left(0.88 \alpha h_{5}(\alpha)\right)^{s} .
$$

Now for $\alpha>1$

$$
h_{5}(\alpha) \leq \lim _{\alpha \rightarrow 1} h_{5}(\alpha) \leq \lim _{\alpha \rightarrow 1} h_{6}(\alpha) \leq h_{6}(1)=\frac{1}{2},
$$


and combining the last two inequalities we produce the required result:

$$
P_{\mathrm{m}}(n) \leq \frac{n}{s !^{2}}\left(0.88 \alpha h_{5}(\alpha)\right)^{s} \leq \frac{n}{s !^{2}}\left(\frac{0.88}{2} \alpha\right)^{s} \leq \frac{n}{s !^{2}}\left(\frac{\alpha}{2}\right)^{s} .
$$

Proof of Remark 2: The proof follows the same lines as the third stage of the proof of Theorem 3. The only difference is that we take the first two terms in the sum of the right hand side of (4.23). Thus instead of (4.22) we receive

$$
h(x) \leq H(x)=2 x^{2 \alpha}\left(1-\frac{2 x^{2}}{3}-\frac{2 x^{4}}{15}\right) .
$$

The maximum point of $H(x)$ turns out to be

$$
x^{*}=\frac{3 \alpha}{(\alpha+1) \sqrt{1+\frac{6 \alpha(\alpha+2)+1}{5(\alpha+1)^{2}}}} .
$$

Calculating $H\left(x^{*}\right)$ and proceeding as in the proof, we obtain the required result.

\section{References}

1. D. Berend and J. Harmse, Expert rule versus majority rule under partial information. Theory and Decision, 35:179-197, 1993.

2. D. Berend and J. Paroush, When is Condorcet's Jury Theorem valid? Social Choice and Welfare, 15:481-488, 1998.

3. D. Berend and L. Sapir, Optimality of the expert rule under partial information. preprint, 2001.

4. S. Berg, Condorcet's Jury Theorem, dependence among jurors. Social Choice and Welfare, 10:87-95, 1993.

5. S. Berg, Condorcet's Jury Theorem revisited. European Journal of Political Economy, 9:437-446, 1993.

6. S. Berg, Indirect voting systems: Banzhaf numbers, majority functions and collective competence. European Journal of Political Economy, 13:557-573, 1997.

7. S. Berg and J. Paroush, Collective decision making in hierarchies. Mathematical Social Sciences, 35:233-244, 1998.

8. P. Boland, Majority systems and the Condorcet Jury Theorem. The Statistician, 38:181-189, 1989.

9. P. Boland, F. Proschan, and Y. Tong, Modelling dependence in simple and indirect majority systems. Journal of Applied Probability, 26:81-88, 1989.

10. N.C. de Condorcet, Essai sur l'application de l'analyse á la probabilité des décisions rendues á la pluralité des voix. Paris, 1785 .

11. M.A. Golberg, An introduction to probability theory with statistical applications. Plenum Press, New York and London, 1984. 
12. M. Gradstein and S. Nitzan, Performance evaluation of some special classes of weighted majority rules. Mathematical Social Science, 12:31-46, 1986.

13. B. Grofman, G. Owen, and S. Feld, Thirteen theorems in search of the truth. Theory and Decision, 15:261-278, 1983.

14. K. Ladha, Information polling through majority rule voting: Condorcet's Jury Theorem with correlated votes. J Econ Behavior Organizat, 26:353-372, 1995.

15. N. Miller, Information, electorates, and democracy: some extensions and interpretations of Condorcet Jury Theorem, in information pooling and group decision making. ed. B. Grofman and G. Owen, Greenwich, CT:JAI Press, 1986.

16. S. Nitzan and J. Paroush, Optimal decision rules in uncertain dichotomous choice situations. International Economic Review, 23:289-297, 1982.

17. S. Nitzan and J. Paroush, A general theorem and eight corollaries in search of a correct decision. Theory and Decision, 17:211-220, 1984a.

18. S. Nitzan and J. Paroush, Partial information on decisional competences and the desirability of the expert rule in uncertain dichotomous choice situations. Theory and Decision, 17:275-286, 1984b.

19. S. Nitzan and J. Paroush, Collective decision making. Cambridge University Press, Cambridge, 1985.

20. J. Paroush, Stay away from fair coins: a correct Condorcet's Jury Theorem. Social Choice and Welfare, 15:15-20, 1998.

21. L. Sapir, The optimality of the expert and majority rules under exponentially distributed competence. Theory and Decision, 45:19-35, 1998.

22. H. Young, Condorcet's Theory of voting. American Political Science Review, 82:1231-1244, 1989. 


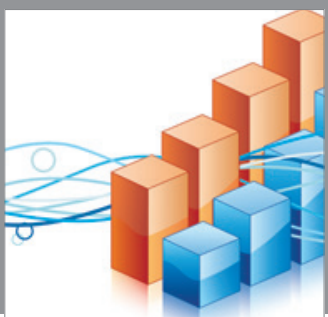

Advances in

Operations Research

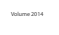

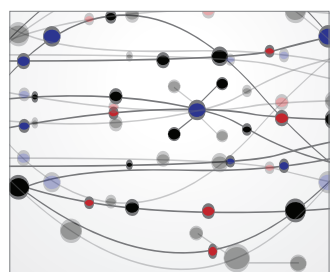

\section{The Scientific} World Journal
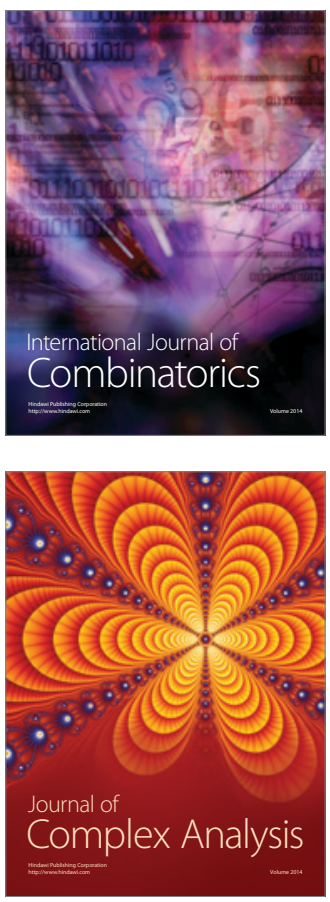

International Journal of

Mathematics and

Mathematical

Sciences
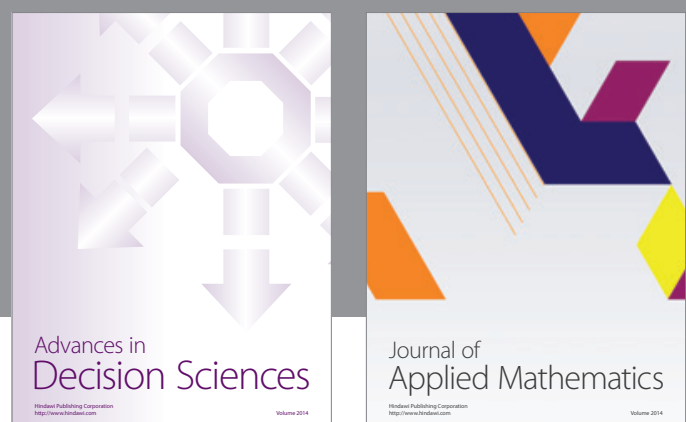

Journal of

Applied Mathematics
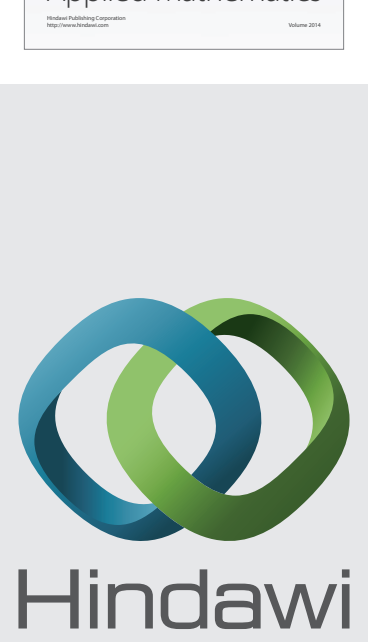

Submit your manuscripts at http://www.hindawi.com
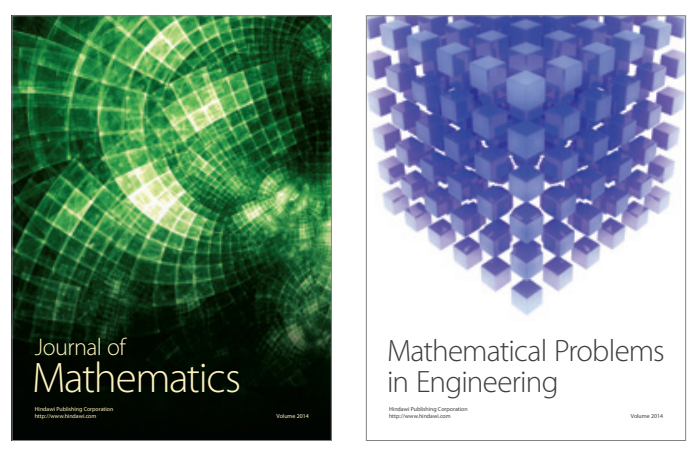

Mathematical Problems in Engineering
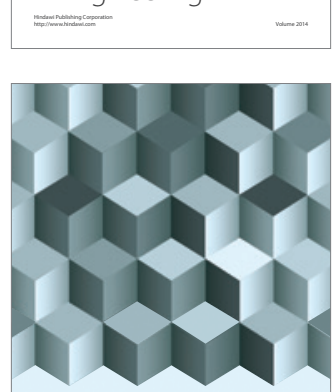

Journal of

Function Spaces
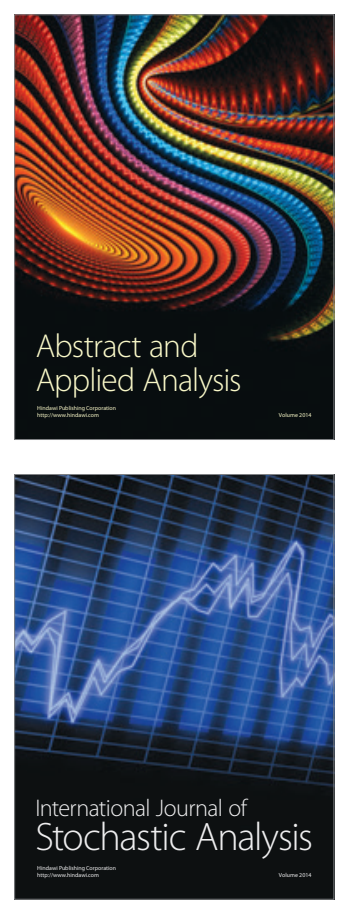

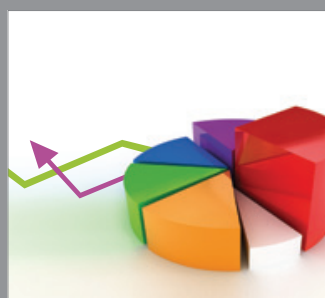

ournal of

Probability and Statistics

Promensencen
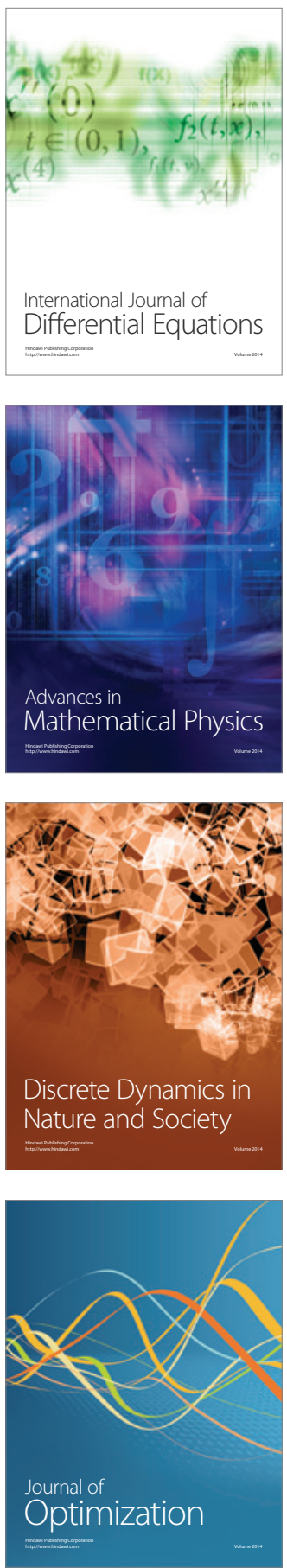